\title{
An improved method for the determination of low-level non-exchangeäble organically bound tritium in biological samples
}

\author{
F. Pointurier, N. Baglan, A. Alanic and R. Chiappini \\ CEA/DAM/DIF/DASE/SRCE, BP. 12, 91680 Bruyères-le-Châtel, France
}

\begin{abstract}
In this paper, the authors present a sensitive method for low level non-exchangeable OBT determination by a combination of a suitable sample treatment, a combustion apparatus for large sized samples and low background liquid scintillation spectrometry, along with a methodology that eliminates any possibility of sample contaminations. Great care must be taken in the measurement of non-exchangeable OBT at environment levels, because many authors have mentioned the opportunities for cross contaminations between samples and contamination by exchange with the laboratory atmosphere. Our analysis are reproducible for low level OBT down to about $1 \mathrm{~Bq} \cdot \mathrm{kg}^{-1}$ of dry material for various biological samples (tree wood, tree leaves, fishes, sediments, mushrooms, seaweeds, wheats, corns, ...) collected in the general environment and near nuclear facilities. As an example, we give the results of non-exchangeable OBT measurements in annual growth rings from two trees sampled near a nuclear facility.
\end{abstract}

\section{INTRODUCTION}

Tritium can be present in vegetation and soil as a constituent of water, organic matter or soil minerals. The tritium in vegetation and soil samples that is easily removed by evaporation at low temperature or by freze-drying is in the form of tritiated water (HTO). Tritium that is associated with organic matter or soil minerals is more difficult to remove from samples and is often designated "organically bound" tritium (OBT).

The OBT of soil or vegetation can be further divided into an exchangeable fraction and a nonexchangeable fraction [1-5]. Exchangeable organically bound tritium is essentially bound to oxygen and nitrogen atoms in compounds of the material. It will rapidly be in equilibrium with the overall aqueous component of the system and so, the free water and the exchangeable OBT will tend to equilibrate rapidly with local atmospheric moisture. By contrast, non-exchangeable organically bound tritium is bound to carbon atoms in the material and, thus, is tightly held to the organic structure.

A special case is wood from tree rings which also retains tritium as a form of OBT, photosynthesised both from HTO in soil around the tree roots at the time of ring formation and from the atmospheric water vapour. Soil water tritium is derived from washout of tritiated vapour in air. Measurements of OBT in annual tree rings will reveal the historical environmental tritium levels and hence make it possible to evaluate the local effect of tritium discharge from nuclear facilities by comparing the tree rings OBT levels in the general environment with those near the facilities [6-9].

Great care must be taken in the measurement of OBT at environment levels. Many authors [5,10-14] have pointed out the opportunities for cross contamination of samples and contamination by exchange with the laboratory atmosphere. Moreover, our laboratory is located not far from a nuclear research centre where atmospheric HTO levels are usually elevated above the normal environment levels that we often ty to measure. We consider that, for proper analyses, the following points must be achieved:

- A combustion unit which can accommodate at least 30 grams of dried sample, and so to deliver at least 10 grams of combustion water to get enough counts for acceptable statistical accuracy.

- A complete removal of the water from the sample, especially after the elimination of the exchangeable OBT. The water may have been contaminated by exposure to the laboratory atmosphere at any stage of the treatment. 
- Removal as complete as possible, of the exchangeable OBT from the material, thanks to a fast and efficient technique, which does not degrade the organic structure of the sample.

- Protection of the sample against exposure to the laboratory atmosphere, before and after drying and combustion, especially after the final drying just before combustion, because the dried material at this stage is very hygroscopic and will pick up water from the laboratory air when exposed during the transfer to the combustion system.

- Proper use of low background liquid scintillation counters, with appropriate blanks for the whole process.

In this paper, the authors describe a sensitive method for low level non-exchangeable OBT determination by a combination of a suitable sample treatment, a large capacity combustion apparatus and low background liquid scintillation spectrometry.

\section{EXPERIMENTAL}

\section{2-1. Sampling}

As there is limited correlation between samples of different types [5,13], it is recommended to sample a limited number of biological species, if possible only one or two species available in the sampled area. It is also advised to sample at the end of the growing period, so that the OBT integration is maximised.

The packaging of the samples is particularly important because it must provide an impervious barrier to atmospheric water vapour, which can contaminate the samples. We recommend to seal each sample in double welded vinyl bags.

\section{2-2. Pre-treatment of the samples: drying, grinding}

The samples must be dried as completely as possible in order to avoid partial destruction of the organic matter by rotting, to eliminate free water of the samples, and, finally, to make the samples suitable for the next stage of the treatment (grinding). The initial drying of the samples can be performed in drying ovens at moderate temperature $\left(40^{\circ} \mathrm{C}\right)$ swept with dry air or, preferentially, by freeze-drying. In this last case, thanks to a custom-built freeze-dryer, it is possible to recover the free water of the sample.

The samples must be reduced to small particles, with average sizes of approximately two millimetres. If the samples are not dry enough, grinding will not be effective. The grinding of the samples is absolutely necessary to perform an efficiently exchangeable OBT elimination.

\section{2-3. Exchangeable OBT elimination and freeze-drying of the samples}

All samples first undergo isotopic exchange with large amounts of tritium free water (deep-drilling water with tritium activity below $0.25 \mathrm{~Bq} . \mathrm{L}^{-1}$ according to electrolytic enrichment measurement) so as to eliminate the exchangeable OBT. The sample, which has first been reduced to small particles, is mixed with a large volume of deep-drilling water (one litre) in a glass container from one to three days with magnetic stirring. The top aperture of each container is carefully blocked with a special waterproof plastic film. Tests have proved that no tritium contamination of the deep-drilling water in such conditions is detectable by liquid scintillation counting with a limit of detection of about $1 \mathrm{~Bq} \cdot \mathrm{L}^{-1}$.

By isotopic exchange, the tritium content of the exchangeable part of the organically bound hydrogen tends to be averaged with the tritium content of hydrogen atoms from the deep-drilling water, which is supposed to be zero. If we assume that the isotopic exchange is complete, the ration of the remaining exchangeable OBT activity after the exchange $\left(A^{\prime}{ }_{E x}-O B T\right)$ on the exchangeable OBT activity before the exchange $\left(A_{E x-T}\right)$ is:

$$
\frac{A_{E x-T}^{\prime}}{A_{E x-T}}=\frac{M_{E x-H}^{\text {sample }}}{M_{E x-H}^{\text {sample }}+M_{H}^{T \text { free water }}} \cong \frac{M_{E x-H}^{\text {sample }}}{M_{H}^{T \text { free water }}}
$$


where $M_{E x-H}^{\text {sample }}$ is the remaining mass after drying of exchangeable hydrogen in the sample and $M_{H}^{T \text { free water }}$ the mass of hydrogen in the tritium free water used for the isotopic exchange, which is equal toaninth of $M^{T \text { free water }}$, the total mass of free water used.

In vegetation samples, we consider roughly that about $30 \%$ of the hydrogen organic atoms are located in exchangeable positions, and that the total hydrogen content in mass is about $6 \%$. Then, with 35 gof dry sample and $1 \mathrm{~kg}$ of tritium free water, the equation (1) becomes, assuming isotopic exchange is complete:

$$
\frac{A_{E x-T}}{A_{E x-T}} \cong \frac{0.06 \times 0.3 \times M^{\text {sample }}}{\frac{1}{9} \times M^{T \text { free water }}} \cong 0.16 \times \frac{M^{\text {sample }}}{M^{T \text { free water }}} \cong \frac{1}{180}
$$

We can thus consider that the remaining exchangeable OBT activity is negligible compared with the nonexchangeable OBT activity, unless the sample had undergone a very strong tritium contamination during one of the preparation steps.

At the end of the isotopic exchange, the water is eliminated simply by filtering the solution inside a glove box supplied with dry air. The wet sample is immediately put inside a quartz boat suitable for combustion. The remaining exchange water is eliminated by freeze-drying. At the end of freeze-drying, the vacuum is broken with argon so as to protect the sample from ambient air, and the sample is immediately introduced into the combustion oven which is swept by a flux of pure and dry oxygen.

\section{Combustion of the samples}

The principle of our combustion apparatus (ERALY, Noisy-le-Sec, France) is relatively close to others arready described by some authors [15-18]. It consists of a furnace with two in-line heating regions and of a guartz tube which crosses the two heating regions (see figure 1 below). In the first region, the sample is held in a quartz boat, of nominal capacity $120 \mathrm{~cm}^{3}$, which is itself enclosed in the quartz tube. The part of the quartz tube which is inside the second heating region contains a catalyst consisting of a mixture of guartz pellets ( $90 \%$ by weight) and cobalt oxide ( $10 \%$ by weight). The temperatures of both regions are independently adjustable. The $1^{\text {st }}$ heating region is mobile. The quartz boat containing the sample is placed just ahead of the $1^{\text {st }}$ heating part, so that the extremity of the sample is heated by the furnace. When the sample starts to ignite, a flame appears at the extremity of the sample and the heating region is noved towards the sample so that the flame moves slowly across the whole sample. By doing this, we ensure slow and complete combustion of the sample, which is completely reduced to ash.

The $2^{\text {nd }}$ heating region is fixed and held at a constant temperature. The combustion gases are swept by a stream of pure oxygen over the catalyst. The combustion gases are oxidised by the catalyst and the water vapour is trapped in the cold trap, cooled at $-25^{\circ} \mathrm{C}$ by a cryostat. Only a small part of the water vapour escapes from the trap and is absorbed by a desiccant trap at the exit of the cold trap. All the combustion parameters (oxygen flux, speed of the mobile heating part, temperatures of the two heating parts and of the cold trap, ...) depend largely on the nature of the sample.

At the end of combustion (sample totally reduced to ash), the piece of glassware containing the frozen combustion water is removed from the combustion apparatus and immediately closed with appropriate caps. The volume of combustion water obtained typically ranges from 6 to $15 \mathrm{ml}$, depending on the type of sample, for a mass of burnt sample of about 35 grams. But the combustion water is not sutable for liquid scintillation counting because of the various impurities also collected in the cold trap. The water has to be purified by a single distillation under atmospheric pressure after $\mathrm{pH}$ adjustment with sodium peroxide $\left(\mathrm{Na}_{2} \mathrm{O}_{2}\right)$. For the distillation we use conventional laboratory glassware. 


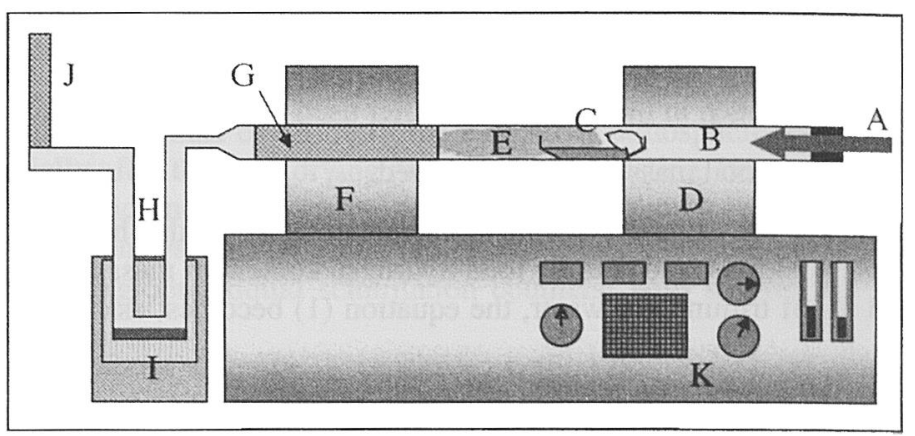

Figure 1. Schematic drawing of our combustion system for biological samples. A: dry and pure oxygen flux; B: quartz tube; C: sample during combustion; D: first heating unit (mobile); E: combustion gases; F: second heating unit (fixed); (fi: cobalt oxide catalyst with quartz bead; $\mathrm{H}$ : "U" tube for water vapour trapping; $\mathrm{I}$ : cold trap; J: dessiccant (silicagel); K: combustion parameters controllers.

\section{2-5. Protection against the risks of contamination and blank measurements}

We prevent possible contamination from airborne tritiated vapour by heating under vacuum for a feiw hours all the glassware used during the experiments. The vacuum is broken with dry argon and the glassware is flushed with dry argon for some minutes before assembling the apparatus.

After the combustion of each sample, we perform a "combustion blank" through the "combustion" of about $15 \mathrm{ml}$ of tritium free water. The recovered solution is neutralised and distilled as well as the combustion water of the sample and finally measured by liquid scintillation counting under similar conditions. We proved that the tritium memory effect of the combustion apparatus is very low: the activity of the combustion water of the "combustion blank" performed after the combustion of a high activity biologic sample is below $1 \%$ of the sample's combustion water activity.

\section{2-6. Tritium activity measurements by liquid scintillation counting}

Once neutralised and purified by distillation, the combustion waters are ready for liquid scintillation measurement. They are mixed with a high efficiency LSC (Liquid Scintillation Counting) cocktal (PACKARD "UltimaGold LLT") in the following proportions: 10 grams of combustion water and $10 \mathrm{~g}$ of LSC cocktail. We use low background and low diffusion polyethylene LSC vials (PACKARD).

Most of the measurements were made with two WALLAC "Quantulus" (Turku, Finland), which have a detection limit of about $1 \mathrm{~Bq} \cdot \mathrm{L}^{-1}$ with 10 grams of water, a 24 hours counting time and a background of about $0.7 \mathrm{cpm}$ in the 50 to 180 channels window. The quenching is corrected thanks toa quenching curve calculated after the measurement of a series of home-made quenched standards.

\section{2-7. Activity and uncertainties calculations}

The specific activity $A$ of the combustion water in $\mathrm{Bq} \cdot \mathrm{L}^{-1}$ is given by the following formula:

$$
A=\frac{1000 \times\left(C_{\text {sample }}-C_{\text {blank }}\right) \times d}{60 \times E \times M}
$$

where $C_{\text {sample }}$ and $C_{\text {blank }}$ are respectively the count rates of the sample and of the blank, expressed in cpm (counts per minute), $E$ is the counting efficiency of the counter (number without dimension, between 0 and 1); $M$ is the mass of measured combustion water inside the counting vial, expressed in grams and $d$ is the density of water, which is assumed to be equal to $1 \mathrm{~g} \cdot \mathrm{mL}^{-1}$.

The uncertainty $I_{A}$ with a confidence level of $95 \%$ is given by the classical propagation error formula:

$$
I_{A}=2 \times \sqrt{\left(\frac{\partial A}{\partial C_{\text {sample }}} \cdot \sigma_{C_{\text {sample }}}\right)^{2}+\left(\frac{\partial A}{\partial C_{\text {blank }}} \cdot \sigma_{C_{\text {blank }}}\right)^{2}+\left(\frac{\partial A}{\partial E} \cdot \sigma_{E}\right)^{2}+\left(\frac{\partial A}{\partial M} \cdot \sigma_{M}\right)^{2}+\left(\frac{\partial A}{\partial d} \cdot \sigma_{d}\right)^{2}}(4)
$$


Assuming that the total counting time $t$ (in minutes) is the same for a sample and its blank and that the relative uncertainty over the density of water is negligible, we obtain:

$$
I_{A}=2 \times A \times \sqrt{\frac{C_{\text {sample }}+C_{\text {blank }}}{t \times\left(C_{\text {sample }}-C_{\text {blank }}\right)^{2}}+\left(\frac{\sigma_{E}}{E}\right)^{2}+\left(\frac{\sigma_{M}}{M}\right)^{2}}
$$

The relative uncertainty over the counting efficiency and the mass of measured solution are respectively to $4 \%$ and $1 \%$.

So as to estimate the significance of a calculated activity, we use the common expressions of the decision threshold $D T$ and of the detection limit $D L$ calculated from the definitions given by M. Neuilly [19]. When $A$ is less than $D T$, the announced result is " $A<D L$ ". When $A$ is more than $D T$, the announced result is " $A \pm I_{A}$ ". With a chosen value of $2.5 \%$ for both risks $\alpha$ and $\beta$, the decision threshold and the detection limit, expressed in Bq. $\mathrm{L}^{-1}$, are given by the following formulas:

$$
D T=2 \times \frac{100}{60 \times E \times M} \times \frac{1}{t} \times\left(1+\sqrt{1+2 \times t \times C_{\text {blank }}}\right) \quad \text { (6) and } D L=2 \times D T \text { (7) }
$$

The specific activity can also be expressed in $\mathrm{Bq}$ per $\mathrm{kg}$ of dry material. However, the hydrogen recovering yield $r_{H}$ through the combustion process cannot be measured precisely because the mass of hydrogen in the samples is not known and because of the lack of certified standard material for OBT measurements. Nevertheless, this yield can be estimated from the combustion of solution of known tritium activity. We take the value of $95 \pm 5 \%$ ( $95 \%$ confidence level). Then, the specific activity $a$ by weight of dry material is given by:

$$
a=\frac{A \times m_{\text {combustion water }}}{r_{H} \times d \times m_{\text {dry material. }}}=\frac{1000 \times\left(C_{\text {sample }}-C_{\text {blank }}\right) \times m_{\text {combustion water }}}{60 \times E \times m_{\text {dry material. }} \times M_{\text {measured water }}}
$$

\section{EXAMPLE OF APPLICATION}

As an example of application of this methodology, we give the non-exchangeable OBT activities of the annual rings of wood sections from two trees sampled near a nuclear facility (see figure 2 below).

The ring number 1 corresponds to the year of sampling. The measurements had been performed on raw wood, without cellulose extraction. The measured activities are very low, below $10 \mathrm{~Bq} \cdot \mathrm{L}^{-1}$ of combustion water, except for the ring number 7 for both trees, which is undoubtedly higher than the others. We can thus consider that this nuclear facility had released an unusual tritium activity six years before the sampling.

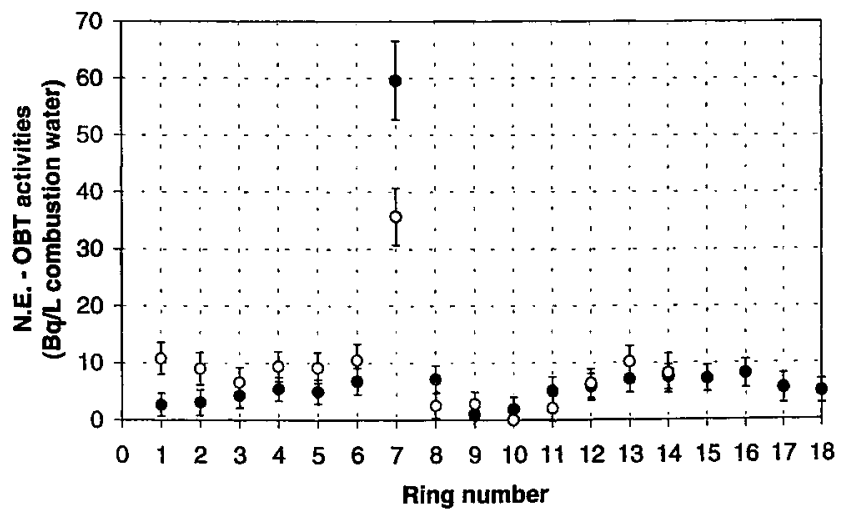

ligure 2. Non-exchangeable OBT activities for a cypress (black circles) and a tamarisk (white circles), sampled in the vicinity All the All the uncertainties are given with a $95 \%$ confidence level.

The annual growth rings are identified with a good confidence thanks to a X-ray microdensitometry technique (INRA, Ardon, France). The individual rings were then carefully chiselled off, cut 
into small-sized pieces and ground with a special grinder dedicated to wood samples. The remainder of the treatment is exactly the same as for other biological samples.

\section{CONCLUSION}

The previous studies realised on OBT measurement allowed to demonstrate the interest of these types of studies and to solve a few fundamental problems, like the possibility to eliminate the exchangeable OBT, and to extract the non-exchangeable $\mathrm{OBT}$ as water measurable by liquid scintillation counting Nevertheless, many authors encountered technical problems, particularly contaminations between samples or by the laboratory atmosphere, and difficulties to explain the discrepancies between the results obtained for different sampled species, or even between samples of the same specie with different local growing conditions.

The authors developed a methodology for non-exchangeable OBT measurement in biological samples which, thanks to an appropriate treatment with special precautions, ensures the protection of the sample against contamination and guarantees the exclusive measurement of non-exchangeable OBT. The quantity of treated samples is enough to give an optimised measurement by low-level liquid scintillation counting, with our best detection limit of about $1 \mathrm{~Bq} \cdot \mathrm{kg}^{-1}$ of dry material. Moreover, thanks to the use of a custom-built freeze dryer and of isotopic exchange in the liquid phase, instead of traditional vapour phase, for the exchangeable tritium elimination, the duration of the whole sample treatment is considerably reduced. This methodology was applied successfully to various biological samples. Some of them presented very low background activities, around a few Bq. $\mathrm{kg}^{-1}$ of dry material.

The non-exchangeable OBT level inside a biological sample is the result of an integration of the local environmental tritium over the functioning period(s) of the sample. Then, the exclusive and reproducible measurement of non-exchangeable OBT allowed by this methodology provides a powerful tool for environmental monitoring. Moreover, it has been proven that it is also possible to obtain retrospective information about past accidental or chronicle tritium releases, even many years after the occurrence of the event.

\section{References}

1. Couchat P., Puard M., Lasceve G; Health Physics 45 (1983) 757-764.

2. Belot Y., Guenot J., Health Physics 47 (1984) 849-855.

3. Sweet C.W., Murphy C.E., Environ. Sci. Technol. 18 (1984) 358.

4. Brown R.M., report AECL-CRNL/INFO-0283 (1988).

5. Brown R.M., report AECL-CRNL/INFO-0499 (1995).

6. Brown R.M, report IAEA/STI/PUB 498, IAEA, Vienna (1979).

7. Kigoshi K., Tomikura Y., Bull. Chem. Soc. Japan 34 (1961) 1738.

8. Kozak K., Rank D., Biro T., Rajner V., Golder F., Staudner F., J. Environ. Radioact. 19 (1993)67.

9. Yamada Y., Itoh M., Kiriyama N., Komura K., Ueno K., J. Radionanal. Nucl. Chem., Articles 130 (1989) 169.

10. Garland J.A., Cox L.C., Water, Air and Soil Pollution 17 (1982) 207-211.

11. Kozak K., Acta Phys. Acad. Scient. Hungaricae 52 (1982) 429-434.

12. Rank D., Kozak K., report IAEA-R-3452-F (1987).

13. Brown R.M., report AECB-3.135.1 (1995).

14. Garland J.A., Ameen M., Health Physics 36 (1979) 35.

15. Lockyer J.F., Lally A.E., The Sci. Of the Tot. Env. 130/131 (1993) 337.

16. Hisamatsu S., Hashimoto T., Takizawa Y., J. Radioanal. Nucl. Chem., Letters 146 no 4 (1990) 245.

17. Bogen D.C., Henkel C.A., White C.G.C., Welford A., J. Of Radioanal. Chem. 13 (1973) 335

18. Takashima Y., Momoshima N., Inoue M., Nakamura Y., Appl. Rad. Isot. 38 no 4 (1987) 255.

19. Neuilly M., CETAMA, "Modélisation et estimation des erreurs de mesure" (TEC DOC Lavoisier, Paris, 1993) pp.374-384. 